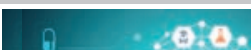 \\ Chemical Education

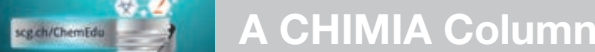 \\ Topics for Teaching: Chemistry in Nature
}

\section{Eloxieren und Färben von Kugelschreiberhülsen}

\section{Rita Oberholzer*}

${ }^{*}$ Korrespondenz: Frau R. Oberholzer, E-Mail: rita.oberholzer@ksimlee.ch, Kantonsschule Im Lee, Rychenbergstrasse 140, CH-8400 Winterthur,

Abstract: Es wird ein motivierendes Experiment zur Anwendung des Eloxal-Verfahrens beschrieben.

Keywords: Elektrolyse · Eloxalverfahren · Kugelschreiber · Redoxreaktionen

Als unedles Metall ist Aluminium grundsätzlich anfällig für Korrosion. Aluminium bildet jedoch an der Oberfläche - im Gegensatz zu Eisen - mit Sauerstoff eine kompakte Oxidschicht. Damit schützt es sich selber vor weiterer Korrosion. Durch Neuaufbau und Verstärkung der Oxidschicht («Eloxalschicht») mittels Elektrolyse kann der Schutz noch um ein Vielfaches erhöht werden. Gleichzeitig wird die Oberfläche härter und sie wirkt elektrisch isolierend. Das Schöne dabei: In die poröse Oxidschicht können Farbstoffe eingelagert werden. Die Farbstoffe dringen zwar wegen ihrer Grösse meist nicht vollständig in die Poren ein. Zur besseren Haftung können die Poren aber am Ende noch «verschlossen» werden.

Im Alltag wird dieses Verfahren Eloxal-Verfahren genannt (Elektrolytische Oxidation von Aluminium). Beinahe alle Werkstücke aus Aluminium sind eloxiert, sichtbar allerdings nur dort, wo sie auch noch eingefärbt sind, so z.B. bei Häuserfassaden, Velorahmen, Verkehrsschildern, Schmuck, Schlüsseln, Karabinern u.a.

Mit einer Gruppe von Schülerinnen und Schülern können Kugelschreiberhülsen aus Aluminium (Caran d'Ache) in einer Doppellektion eloxiert und eingefärbt werden (Abb. 1). Die individuell gestalteten Produkte sind täglich nutzbar, aber auch als Geschenke sehr beliebt. Vor Weihnachten wird deshalb an unserer Schule auch in der Freizeit eloxiert und gefärbt. Alternativ lassen sich Aluminium-Sparschäler eloxieren und einfärben.

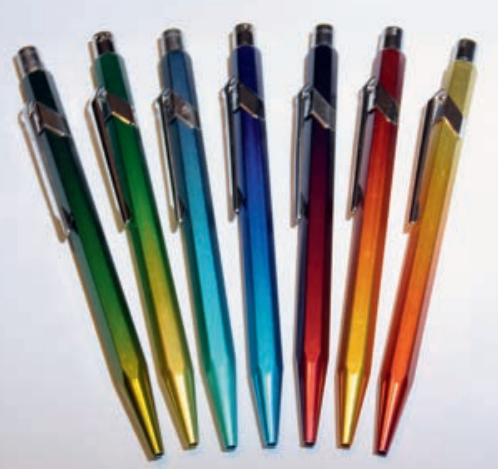

Abb. 1. Beispiele von Kugelschreibern und Sparschälern
Insgesamt sind vier Arbeitsschritte notwendig:

- Reinigung (natürliche Oxidschicht entfernen)

- Eloxieren (neue Oxidschicht aufbauen)

- Färben (Farbstoff einlagern)

- Sealen (Poren verschliessen)

Alle benötigten Lösungen können dutzende Male verwendet werden, bevor sie «aufgefrischt» oder entsorgt werden müssen; es entstehen also praktisch keine Chemikalienabfälle.

Reaktionsmässig steht die Elektrolyse im Zentrum, je nach Wissensstand der Schülerinnen und Schüler kann aber auch auf den Reinigungsprozess eingegangen werden.

\section{Reaktionen beim Eloxieren}

Reduktion: $\quad 6 \mathrm{H}_{3} \mathrm{O}^{+}+6 \mathrm{e}^{-} \rightarrow 3 \mathrm{H}_{2}(\mathrm{~g})+6 \mathrm{H}_{2} \mathrm{O}$

Oxidation $\quad 2 \mathrm{Al} \rightarrow 2 \mathrm{Al}^{3+}+6 \mathrm{e}^{-}$

Zwischentotal: $\quad 2 \mathrm{Al}+6 \mathrm{H}_{3} \mathrm{O}^{+} \rightarrow 2 \mathrm{Al}^{3+}+3 \mathrm{H}_{2}(\mathrm{~g})+6 \mathrm{H}_{2} \mathrm{O}$

Die entstehenden Aluminiumionen reagieren sofort $\mathrm{zu}$ Aluminiumoxid weiter:

$$
\begin{array}{ll} 
& 2 \mathrm{Al}^{3+}+9 \mathrm{H}_{2} \mathrm{O} \rightarrow \mathrm{Al}_{2} \mathrm{O}_{3}(\mathrm{~s})+6 \mathrm{H}_{3} \mathrm{O} \\
\text { Gesamtreaktion: } & 2 \mathrm{Al}+3 \mathrm{H}_{2} \mathrm{O} \rightarrow \mathrm{Al}_{2} \mathrm{O}_{3}(\mathrm{~s})+3 \mathrm{H}_{2}(\mathrm{~g})
\end{array}
$$

Damit genügend Zeit für das Färben bleibt, werden bei uns an der Schule die Apparaturen und Chemikalien ausnahmsweise bereitgestellt. Die bei den einzelnen Arbeitsschritten erwähnten Zeiten müssen unbedingt eingehalten werden, damit bei der Elektrolyse eine regelmässige Oxidschicht aufgebaut werden kann. Die Hülsen dürfen nach der Reinigung nicht mehr mit den Fingern berührt werden, bis sie fertig gefärbt sind. Es ist deshalb wichtig darauf zu achten, dass die Hülsen am Al-Draht gut halten.

\section{Arbeitsanleitung}

\section{Sicherheit}

Schwefelsäure, Salpetersäure und Natronlauge sowie deren Aerosole wirken ätzend, Reinigung und Elektrolyse werden deshalb im Abzug durchgeführt, mit Schutzbrille und Handschuhen.

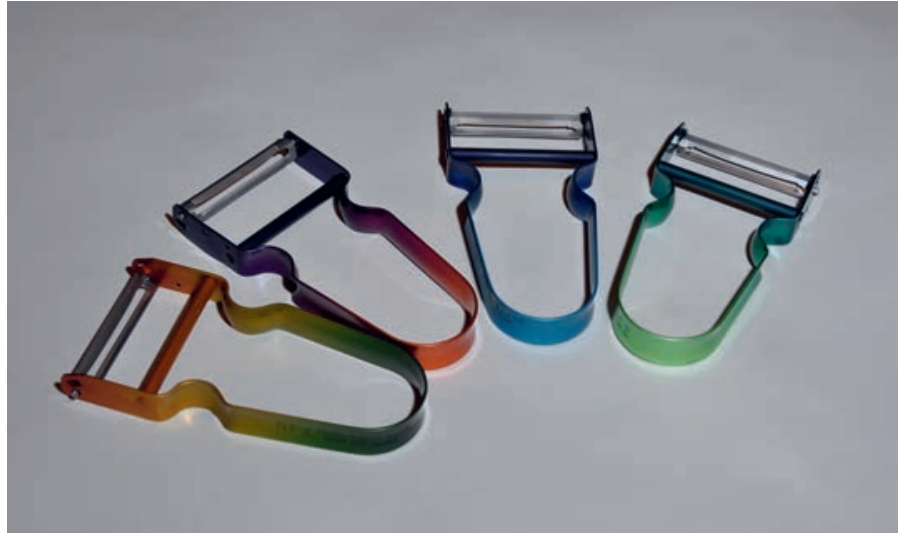




\section{Material und Chemikalien (siehe Hinweise) Reinigung der Hülse}

- Hülse auf den Al-Draht stecken und leicht anklopfen

- zwei Hülsen in einen Halter einspannen

- Becherglas 1 L mit Leitungswasser füllen

- Hülsen in bereitstehende Natronlauge tauchen 1 Min.

- Lösung ausfliessen lassen, Hülsen ins Becherglas mit Wasser stellen und unter fliessendem Wasser spülen 2 Min.

- Hülsen in bereitstehende Salpetersäure tauchen $15 \mathrm{Sek}$.

- unter fliessendem Wasser spülen 2 Min.

- Hülsen innen und aussen mit entmineralisiertem Wasser spülen

\section{Elektrolyse}

Halterung in die bereitstehende Elektrolyseapparatur einspannen und darauf achten, dass die Hülsen vollständig in die Schwefelsäure eintauchen (Abb. 2, 3). Das Kabel vom Pluspol des Netzgerätes wird mit der Halterung verbunden, das Kabel vom Minuspol mit einer Krokodilklemme mit dem Aluminiumblech. Bei voller Spannung wird der Strom auf 1.6 Ampere für zwei Hülsen eingestellt. Elektrolysedauer: 30 Minuten

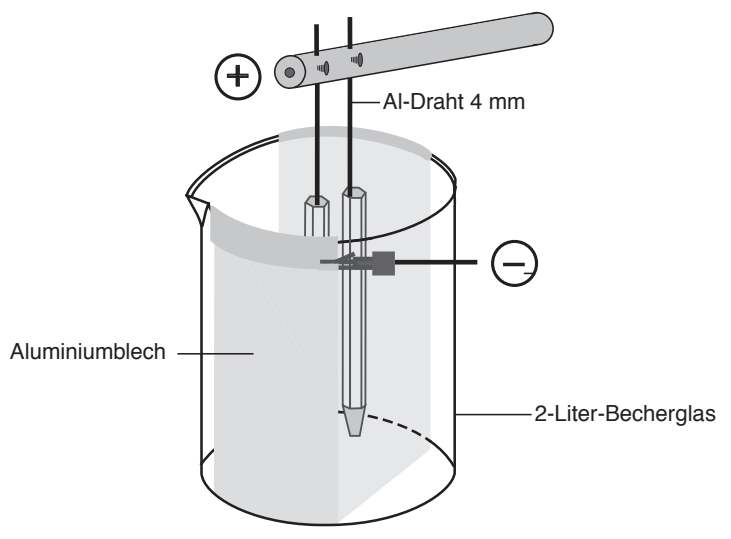

Abb. 2. Apparatur für die Elektrolyse

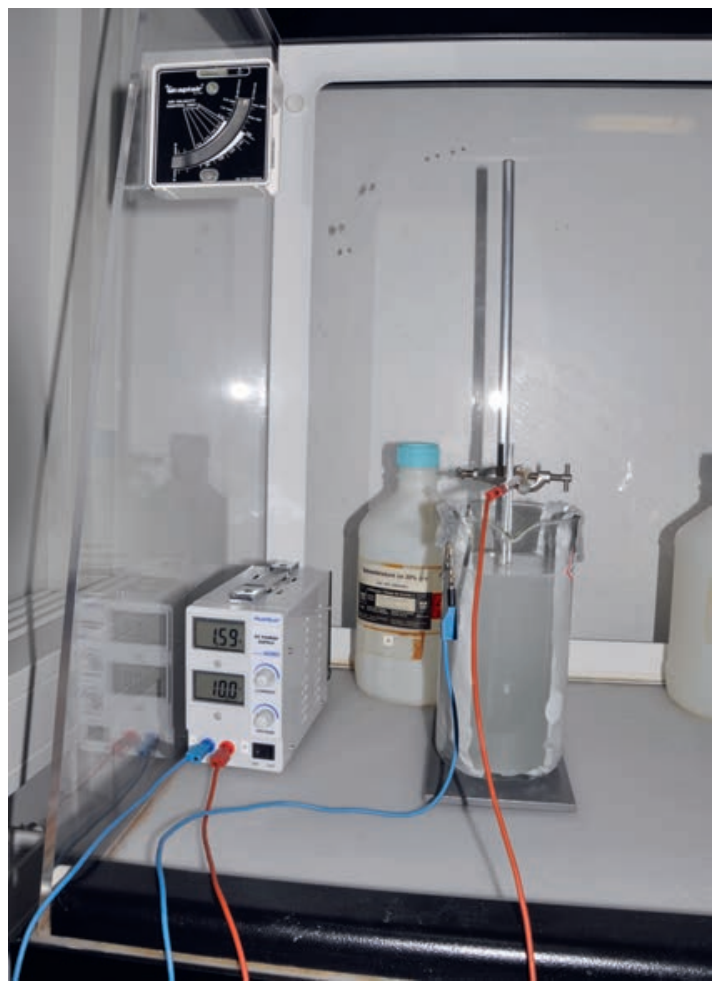

Abb. 3. Elektrolyse
Nach der Elektrolyse die Hülsen erneut unter fliessendem Wasser mindestens 5 Minuten spülen und anschliessend mit entmineralisiertem Wasser abspritzen. Nun können die Hülsen, noch immer am Al-Draht befestigt, einzeln weiterbearbeitet werden.

\section{Färben}

Um regelmässige Farbübergänge zu erhalten, sollten die folgenden Punkte beachtet werden:

- Die Kugelschreiberhülse muss immer nass gehalten werden. Beim Färben müssen also Hülsenteile, die nicht in die Farbe tauchen, regelmässig mit entmineralisiertem Wasser abgespritzt werden.

- Damit die Farbübergänge fliessend werden, muss die Eintauchtiefe ständig variiert werden. Da die Farben unterschiedlich schnell aufziehen, muss der Prozess genau beobachtet werden. Es lohnt sich, die Hülse zuerst nur kurz einzutauchen.

- Vor dem Farbwechsel muss die Hülse aussen und innen mit entmineralisiertem Wasser abgespritzt werden.

- Die Spitze wird zuerst gefärbt. Sobald sie genügend Farbe aufgenommen hat, kann der Schreiber an der Spitze gehalten (Handschuhe tragen!) und umgekehrt in die Farbe getaucht werden.

\section{"Sealen» (Lösung siehe Bezugsquellen)}

Zur Verdichtung der Poren wird die Hülse anschliessend mindestens 5 Minuten in kochendes, sealsalzhaltiges Wasser getaucht. Der Al-Draht wird dazu mit dem Namen der Schülerin/ des Schülers beschriftet.

\section{Entsorgung}

Die Farbstoff- und die Schwefelsäure-Lösungen sowie die Reinigungsbäder (Natronlauge und Salpetersäure) werden wiederverwendet. Die verschiedenen Spülwässer können in den Ausguss gegeben werden.

\section{Hinweise}

\section{Geräte/Glaswaren/Chemikalien pro Arbeitsgruppe}

Netzgerät, Stativ, Muffe, Spezialaufhängung, Krokodilklemme, 2 Laborkabel, Becherglas $2000 \mathrm{~mL}$, Becherglas $1000 \mathrm{~mL}$, grosses Aluminiumblech als Kathode (Minuspol), 1-2 Kugelschreiberhülse(n); Al-Draht, 2 Liter Schwefelsäure ca. 20\%

\section{Zusätzliche Geräte/Chemikalien für die ganze Klasse}

Becherglas $1000 \mathrm{~mL}$ mit Natronlauge, $\mathrm{c}(\mathrm{NaOH})=4-5 \mathrm{~mol} / \mathrm{L}$; Becherglas $1000 \mathrm{~mL}$ mit Salpetersäure, ca. 30\%; Becherglas $3000 \mathrm{~mL}$ mit Sealsalz-Lösung, 5g Nickel(II)-acetat/Liter oder Anodal SH-1 (siehe Bezugsquellen); verschiedene Bechergläser 1000 mL (idealerweise hohe Form) für Farbstofflösungen; pro Farbstofflösung ein Heizrührgerät (notfalls Heizplatte, gefärbt wird bei $65^{\circ} \mathrm{C}$ ); neben jeder Farbstofflösung ein Becherglas 500$1000 \mathrm{~mL}$ für Spülwasser

\section{Hinweise zum Eloxieren}

- Grundsätzlich werden pro $\mathrm{dm}^{2}$ Oberfläche (Innenflächen nicht vergessen!) 1,5 Ampere benötigt. Für zwei Kugelschreiberhülsen resultieren 1.6 Ampere, ebenso für einen Sparschäler.

- Die Schwefelsäure erwärmt sich beim Eloxieren. Die Temperatur darf gemäss eigenen Tests $24{ }^{\circ} \mathrm{C}$ nicht übersteigen, ideal sind $17-21^{\circ} \mathrm{C}$. Bei höheren Temperaturen entsteht eine Eloxalschicht, in der die Farbstoffe nicht richtig eingelagert werden.

- Beim Eloxieren von zwei Kugelschreiberhülsen steigt die Temperatur im 2-Liter-Becherglas um ca. 4-5 ${ }^{\circ} \mathrm{C}$ an. Die Starttemperatur darf also $20{ }^{\circ} \mathrm{C}$ keinesfalls übersteigen. 
Im Sommer, bei grösseren Oberflächen oder bei mehreren Eloxiervorgängen nacheinander muss die Schwefelsäure allenfalls in einem Wasserbad gekühlt werden.

\section{Bezugsquellen}

Eloxalfarben und Anodal SH-1: Musterpackungen sowie Hinweise zur Herstellung der Farblösungen können bei Clariant in Muttenz bezogen werden, ebenso das Anodal SH-1 für das Sealbad: Tel. +41 (0)61 4695111

Kugelschreiber erhältlich bei Firmen, die Werbegeschenke herstellen, z.B. Kaiser Promotion AG, Achslenstrasse 15, 9016 St. Gallen, Tel. +41 (0)71 3139911 (Preis Fr. 8.- bis Fr. 9.-; dieser wird bei uns den Schülerinnen und Schülern verrechnet).

Sparschäler Rex können bei der Migros bezogen werden; der Augenausstecher muss entfernt werden.

Aluminium (Draht und Blech bzw. dicke Folie) z.B. bei Häuselmann in Dietlikon (ZH), +41 (0)44 8351611

\section{Spezialaufhängung}

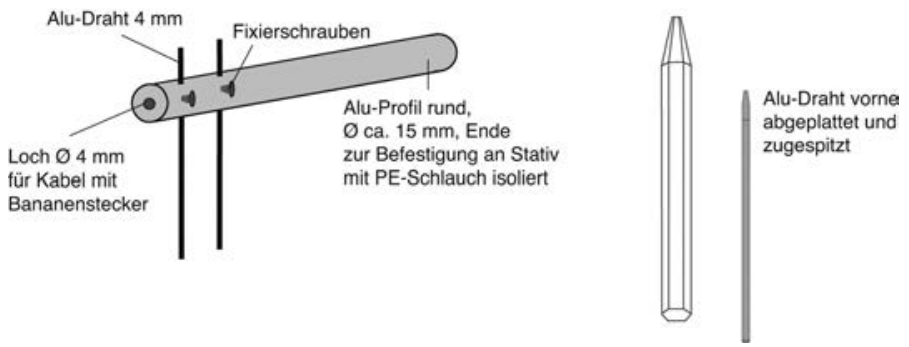

Abb. 4. Spezialaufhängung

\section{Dank}

Ich danke unserem ehemaligen Laboranten Karl Ehrensperger für seine Mitarbeit bei der Entwicklung der Aufhängevorrichtung und für Hinweise zu den Eloxierbädern. 